\title{
Restricting night-time eating reduces daily energy intake in healthy young men: a short-term cross-over study
}

\author{
James D. LeCheminant ${ }^{1 *}$, Ed Christenson ${ }^{2}$, Bruce W. Bailey ${ }^{3}$ and Larry A. Tucker ${ }^{4}$ \\ ${ }^{1}$ Department of Exercise Sciences, Brigham Young University, 269 SFH, Provo, UT 84602, USA \\ ${ }^{2}$ Department of Exercise Sciences, Brigham Young University, 116 RB, Provo, UT 84602, USA \\ ${ }^{3}$ Department of Exercise Sciences, Brigham Young University, 267 SFH, Provo, UT 84602, USA \\ ${ }^{4}$ Department of Exercise Sciences, Brigham Young University, 237 SFH, Provo, UT 84602, USA \\ (Submitted 5 October 2012 - Final revision received 8 March 2013 - Accepted 25 March 2013 - First published online 23 May 2013)
}

\begin{abstract}
Few experimental data are available to support the notion that reducing night-time eating changes total daily energy intake (EI) or body weight in healthy adults. The present study primarily examined the short-term effect of night eating restriction (NER) on daily EI in healthy young men. It secondarily examined body weight and moods associated with NER. Using a cross-over design, twenty-nine men $\left(20.9(\mathrm{SD} 2.5)\right.$ years; $\left.24.4(\mathrm{SD} 2.5) \mathrm{kg} / \mathrm{m}^{2}\right)$ initiated a 2-week NER intervention (elimination of EI from 19.00 to 06.00 hours) and a 2-week control condition, counterbalanced and separated by a 1-week washout period. EI and macronutrient intake were assessed using computerised, multiple-pass $24 \mathrm{~h}$ food recalls, body weight via a digital scale and mood using the Profile of Mood States survey. Of the twenty-nine participants, twenty-seven (93\%) completed all aspects of the study. During the NER condition, the participants consumed less total energy per $\mathrm{d}$ than during the control condition $(10125 v \cdot 11146 \mathrm{~kJ} / \mathrm{d} ; F=6 \cdot 41 ; P=0 \cdot 018)$. During the NER condition, no energy was reported consumed between 19.00 and 06.00 hours; however, during the control condition, the energy intake of participants was 2920 (SD 1347) kJ/d between 19.00 and 06.00 hours. There was a significant difference in weight change between the NER $(-0.4(\mathrm{SD} 1.1) \mathrm{kg})$ and control $(+0.6(\mathrm{SD} 0.9) \mathrm{kg})$ conditions $(F=22.68 ; P<0.001)$. Differences in total mood score or mood subscales between the NER and control conditions were not apparent $(P>0.05)$. These findings provide support for NER decreasing short-term EI in healthy young men.
\end{abstract}

\section{Key words: Night: Night eating: Night eating restriction: Energy intake: Obesity and weight management}

Unhealthy dietary patterns have been linked to gradual weight gain in adults over time ${ }^{(1)}$. However, young adults appear to be at a particular risk for poor dietary habits and significant weight gain ${ }^{(2,3)}$ and, thus, warrant particular attention. To prevent weight gain, some researchers have focused on sustainable lifestyle modifications including a small-changes approach that emphasises increased physical activity and reduced energy intake ${ }^{(4,5)}$. In addition, modest improvements in environmental conditions that promote excessive eating ${ }^{(6)}$ may attenuate the gradual weight gain experienced by many adults across their lifespan ${ }^{(5)}$.

It has been suggested that a greater volume of food is consumed as the day progresses ${ }^{(7)}$. In addition, the evening hours typically result in increased television viewing, which has been associated with increased snacking and food consumption $^{(8)}$. Thus, the evening hours probably represent a high-risk environmental condition for overeating $^{(9)}$ in many adults.
Interestingly, anecdotal weight management recommendations often include advice to limit food consumption at night. This is logical; however, there are surprisingly few experimental data to support the notion that a reduction in or elimination of eating after a certain time of night results in a lower daily energy intake or effective weight management in healthy adults without the night eating syndrome ${ }^{(10,11)}$ or those who do not work the night-time shift ${ }^{(12)}$.

To provide initial experimental data and proof of concept, the present study primarily examined the short-term trends in energy intake and macronutrient intake resulting from the elimination of night-time eating. Young adult men were chosen in the present study as many are likely to engage in unhealthy behaviours ${ }^{(13)}$ and could potentially benefit from the intervention. The present study secondarily examined whether or not there was a trend in body weight change and whether there were differences in mood as a result of this intervention. We hypothesised that night-time eating

Abbreviations: NER, night eating restriction; POMS, Profile of Mood States; VAS, visual analogue scale.

*Corresponding author: J. D. LeCheminant, fax +1 801422 0555, email lecheminant@byu.edu 
restriction would be associated with a lower total daily energy intake compared with the control condition.

\section{Methods \\ Participants}

The present study was conducted according to the guidelines laid down in the Declaration of Helsinki, and all procedures involving human subjects were approved by the Institutional Review Board of the university. Written informed consent was obtained from all participants. The participants were recruited through fliers, posted advertisements, word of mouth and Facebook. Each participant gave his consent to participate in the study and was given a signed copy of the consent form for future reference. As part of the recruitment process, all interested participants were screened, via telephone, to ensure that they met the study criteria given below.

The participants of the present study were unmarried men, 18-26 years of age, healthy, and non-smokers or drinkers and had their weight stable for the previous month. Participant characteristics are given in Table 1. Each participant was screened for the night eating syndrome, on the basis of the night eating syndrome characterisation proposed by Stunkard et al. ${ }^{(10,14)}$, and excluded if the participant reported that he (1) had no appetite for breakfast, (2) had evening hyperphagia (greater than half of his daily energy intake after 19.00 hours) or (3) had trouble getting to sleep or staying asleep three or more days per week, all for the previous 3 months. Nevertheless, the participants were not required to be regular breakfast eaters. Additional exclusion criteria were as follows: current dieting or extreme dietary practices; the presence of an eating disorder (anorexia, bulimia or binge-eating); digestive disorders; a metabolic disease that could affect energy balance (e.g. diabetes mellitus, cancer or heart disease). As exercise may influence food-seeking behaviour ${ }^{(15)}$, the participants were also excluded for excessive exercise training (vigorousintensity physical activity $>4 \mathrm{~d}$ /week and 30 min per session), participation in college athletics or any elite sports, or inability to exercise at a moderate-intensity level.

Using a cross-over design, twenty-nine participants initiated a 2-week night eating restriction (NER) intervention (elimination of energy intake from 19.00 to 06.00 hours) and a 2 -week control condition separated by a 1 -week washout period. The order was counterbalanced with half of the participants completing the NER intervention first and the other half completing the control condition first. The order of conditions was determined on the basis of the order of recruitment. The time of 19.00 hours was chosen as many

Table 1. Participant characteristics at baseline

(Mean values and standard deviations, $n$ 29)

\begin{tabular}{lcr}
\hline Variables & Mean & SD \\
\hline Age (years) & 20.9 & 2.5 \\
BMl (kg/m $\left.{ }^{2}\right)$ & 24.4 & 2.5 \\
Body fat $(\%)$ & 21.3 & 7.1 \\
Body weight $(\mathrm{kg})$ & 77.3 & 10.0 \\
\hline
\end{tabular}

individuals may have eaten their evening meal by this time and because the night eating syndrome has previously been described as excessive food consumption after 19.00 hours ${ }^{(16)}$. The following outcomes were assessed during each condition: energy intake and macronutrient intake via computerised, multiple-pass $24 \mathrm{~h}$ food recalls; pre-breakfast hunger level using visual analogue scales (VAS); body weight; mood profile.

\section{Procedures}

After the initial phone screening, interested and qualifying participants presented at the Human Performance Laboratory to reconfirm that they fit within the parameters of the study, to discuss the required study protocol and to undergo body composition testing. In addition, at this time, each participant was trained by a member of the research staff on how to properly complete a computerised, multiple-pass $24 \mathrm{~h}$ food recall by completing one for the previous day. Furthermore, to determine the pre-study dietary intake, the participants were instructed to complete two additional $24 \mathrm{~h}$ food recalls during the next week (one weekday and one weekend day), determined at random, and were then scheduled for their next visit a week later. Each participant was asked to go about his usual schedule and normal lifestyle patterns during this 'lead-in' week.

On the last day of the lead-in week, each participant presented again to the Human Performance Laboratory where he received instruction about the expectations and protocol of the first condition (NER or control) and reviewed again as to how to properly complete the computerised, multiplepass $24 \mathrm{~h}$ dietary recalls. The participants were also weighed, completed a mood survey and were scheduled for their next visit a week later.

At the end of the first and second weeks of each condition, the participants presented again to the Human Performance Laboratory to be weighed and turn in their pre-breakfast hunger VAS (see below). Additionally, after the second week of each condition, a post-condition mood survey was completed. The protocol for each condition was identical except that the participants were instructed according to the current condition.

Night eating restriction condition. For the NER condition, the participants were instructed to avoid consumption of all food or beverages that included any energy (kJ) from 19.00 to 06.00 hours for two consecutive weeks. The consumption of water after 19.00 hours was considered acceptable and preferable. There were no additional instructions or recommendations on the amount or type of food consumed during the daytime hours of 06.00 to 19.00 hours. There were also no recommendations or restrictions on their general lifestyle patterns. To increase compliance, each participant was called $3 \mathrm{~d} /$ week to report on whether or not he had adhered to the NER protocol and to encourage further compliance.

Control condition. During the control condition (2 weeks), the participants were encouraged to follow their normal prestudy daily lifestyle and eating patterns. There were no other instructions or recommendations provided for this period. 
Washout period. During the washout period (1 week) between conditions, the participants were asked to resume their normal lifestyle patterns. The half of the participants who completed the NER condition first were encouraged to resume their pre-study dietary routine. No other instructions or recommendations were provided for this period.

\section{Measurements}

Energy intake and macronutrient intake. Energy intake and macronutrient intake were assessed with a goal of three separate days (two weekdays and one weekend day) during the lead-in week, each week of the NER condition and each week of the control condition using the Automated SelfAdministered 24-Hour system used by the National Cancer Institute. The Automated Self-Administered 24-Hour is a multiple-pass $24 \mathrm{~h}$ recall that determines the amount, type and time of food and beverage consumption. A multiple-pass $24 \mathrm{~h}$ recall is often preferred as a valid and reliable measure of energy intake and macronutrient intake ${ }^{(17)}$. Its most significant advantages are: (1) low participant burden compared with other measures, such as weighed food records, and (2) relatively non-reactive compared with other dietary assessment methods ${ }^{(17)}$. To further reduce the likelihood of reactivity, dietary assessment days were determined randomly and the participants were not informed of the days on which they would complete a $24 \mathrm{~h}$ recall until the evening hours. Subsequently, each participant was asked to complete the multiple-pass recall as soon as possible the following day, preferably early in the morning.

To ensure that the energy and macronutrient data were reliable and represented the normal diet of the participants during the 2-week control condition, a 1-week lead-in period was completed before the intervention, as noted previously. There was no statistical difference in energy intake $(F=0 \cdot 32$; $P=0.578)$, carbohydrate intake $(F=0 \cdot 10 ; P=0.757)$, fat intake $(F=1.30 ; P=0.265)$ or protein intake $(F=0.56 ; P=0.462)$ between the lead-in period and the 2 -week control condition.

Body composition measures. Body weight and composition were assessed during the lead-in week for descriptive purposes. In addition, body weight was assessed at the beginning and end of each condition. Body weight was obtained using a digital scale (Tanita) accurate to $\pm 0.01 \mathrm{~kg}$ with the participants being barefoot and wearing a standardised bathing suit. Height was measured using a stadiometer (Seca), and BMI was calculated as $\mathrm{kg} / \mathrm{m}^{2}$. Body composition was assessed using dual-energy X-ray absorptiometry, which is considered a valid and reliable measure of body composition $^{(18,19)}$.

Pre-breakfast hunger level. Pre-breakfast hunger level was assessed each day during the second week of each condition. The participants rated their hunger level before eating breakfast using a VAS. A VAS is often used to rate subjective feelings of hunger or appetite ${ }^{(20,21)}$. The VAS included the question 'How hungry do you feel right now?' above a line with the words 'Not at All' on the left side of the scale and 'Extremely' on the right side of the scale. The participants were asked to place a vertical slash along the continuum that best described their hunger level at that moment.

Mood. Mood was assessed using the Profile of Mood States (POMS) scale at the beginning and end of each condition. The POMS survey includes sixty-five questions and has been validated previously ${ }^{(22)}$. In addition to the total mood score, the POMS scale includes six subscales: tension; depression; anger; vigour; fatigue; confusion. The POMS scale ranges from 1 (not at all) to 5 (extremely) to address feelings such as how friendly, tense, angry, confused, shaky, peeved, energetic, relaxed, uneasy, exhausted, cheerful, etc. a person feels at that moment ${ }^{(22)}$.

\section{Statistical analyses}

The statistical software package PC-SAS (version 9.3; SAS Institute, Inc.) was employed for all statistical analyses. The level of significance was set at 0.05 for all statistical tests. Descriptive statistics (mean, standard deviation, etc.) are reported for all dependent measures. The mixed-model procedure in SAS was used to detect differences between conditions for all variables of interest (energy intake and macronutrient intake, body weight change, VAS and POMS scale). To account for individual variability, participant was included in the model as a random term. In addition, baseline (lead-in period) energy or macronutrient data were used as a control variable and the order of conditions was used as a control variable. To determine daily energy intake and macronutrient intake, data from the $24 \mathrm{~h}$ recalls across each 2 -week intervention were averaged by person and are reported in Table 2 (macronutrients as a percentage of energy intake). Night eating was determined as energy intake between 19.00 and 06.00 hours. As the POMS scale was completed by each participant pre and post for each condition, the results section presents the condition $\times$ time interaction for total mood score and for each individual mood subscale.

\section{Results}

At baseline, the participants tended to be young (20.9 (SD 2.5) years), of normal weight $\left(24.4(\mathrm{SD} 2.5) \mathrm{kg} / \mathrm{m}^{2}\right)$ and with a body fat percentage of 21.3 (SD $7 \cdot 1) \%$. Of the twenty-nine participants who initiated the study, twenty-seven (93\%) completed all aspects of the study. The two non-completers cited withdrawal for personal reasons and inability to comply with the protocol due to travel. Furthermore, the participants self-reported following the NER protocol on 93.6 (SD 5.6) \% (approximately thirteen out of fourteen) of the prescribed days.

During the NER condition, the participants consumed less daily average energy $(10125 v .11146 \mathrm{~kJ} / \mathrm{d})$ than during the control condition $(F=6.41 ; P=0.018$; Table 2$)$ and significantly less dietary fat $(95 v .110 \mathrm{~g} / \mathrm{d})$ than during the control condition $(F=7.53 ; \quad P=0.011)$. Carbohydrate and protein intakes $(\mathrm{g} / \mathrm{d})$ were also reduced during the NER condition but did not reach statistical significance, though carbohydrate intake $(\mathrm{g} / \mathrm{d})$ trended towards significance $(F=3 \cdot 87 ; P=0 \cdot 060)$. When carbohydrate, fat and protein intakes were expressed as a percentage of energy intake, there were no statistical 
Table 2. Energy intake and macronutrient intake as a percentage of energy by condition*

(Mean values and standard deviations)

\begin{tabular}{|c|c|c|c|c|c|c|c|c|c|}
\hline & \multicolumn{2}{|c|}{$\begin{array}{c}\text { Lead-in dietary } \\
\text { intake }(n 27) \dagger\end{array}$} & \multicolumn{2}{|c|}{$\begin{array}{c}\text { Control } \\
\text { condition ( }(n 27)\end{array}$} & \multicolumn{2}{|c|}{$\begin{array}{c}\text { Night-time } \\
\text { restriction ( }(\text { 27) }\end{array}$} & \multirow[b]{2}{*}{ Differenceł } & \multirow[b]{2}{*}{$F \S$} & \multirow[b]{2}{*}{$P \S$ ๆ } \\
\hline & Mean & SD & Mean & SD & Mean & SD & & & \\
\hline Energy (kJ) & 10917 & 3529 & 11146 & 3106 & 10125 & 3217 & -1021 & $6 \cdot 41$ & 0.018 \\
\hline Carbohydrate (\% of total energy) & $49 \cdot 2$ & 7.9 & $48 \cdot 7$ & $5 \cdot 3$ & $49 \cdot 1$ & $5 \cdot 3$ & 0.6 & 0.17 & 0.687 \\
\hline Fat (\% of total energy) & $35 \cdot 3$ & $5 \cdot 6$ & $37 \cdot 0$ & $4 \cdot 1$ & 35.9 & 3.7 & $-1 \cdot 1$ & 1.40 & 0.248 \\
\hline Protein ( $\%$ of total energy) & $16 \cdot 4$ & $4 \cdot 2$ & $15 \cdot 4$ & $2 \cdot 4$ & $16 \cdot 0$ & $2 \cdot 9$ & 0.6 & 0.99 & 0.329 \\
\hline
\end{tabular}

* Macronutrient percentages are estimates and were determined by multiplying the number of grams of the macronutrient by $16.736 \mathrm{~kJ}$ per $\mathrm{g}$ of carbohydrate $37.656 \mathrm{~kJ}$ per $\mathrm{g}$ of fat, and $16.736 \mathrm{~kJ}$ per g of protein.

† Lead-in dietary intake represents dietary intake during the week before the first experimental condition.

‡Difference = night eating restriction - control condition.

$\S F$ and $P$ represent the difference between the night eating restriction and control conditions with statistical control of the lead-in value for each macronutrient and the order of completion of the condition.

I There was no statistical difference between the lead-in dietary intake and the control condition $(P>0.05)$.

differences ( $P>0.05$; Table 2). During the control condition, the energy intake of participants was 2920 (SD 1347) kJ/d between 19.00 and 06.00 hours, while they did not report consuming any energy between 19.00 and 06.00 hours during the NER condition.

The average weight change during the NER condition was $-0.4(\mathrm{SD} 1 \cdot 1) \mathrm{kg}$ and during the control condition was +0.6 (sD 0.9) $\mathrm{kg}(F=22 \cdot 68 ; P<0.001)$. The total BMI change during the NER condition was $-0 \cdot 1(\mathrm{SD} 0 \cdot 3) \mathrm{kg} / \mathrm{m}^{2}$ and during the control condition was $+0 \cdot 2(\mathrm{sD} 0 \cdot 3) \mathrm{kg} / \mathrm{m}^{2}(F=22 \cdot 73 ; P<0 \cdot 001)$.

The VAS revealed that the participants experienced greater hunger upon waking during the NER condition than during the control condition. On a scale of $0-10$ (10 being most hungry), the participants reported an average pre-breakfast hunger value of 5.7 (SD 1.5) during the NER condition and that of 4.8 (SD 1.1) during the control condition $(F=7 \cdot 82$; $P=0 \cdot 001)$.

Change in mood and its six subscales are given in Table 3. There was no pre/post-change for total mood score or its subscales within the NER and control conditions $(P>0.05)$. A condition $\times$ time interaction for total mood disturbance was not observed $(F=0.47 ; P=0.494)$. Likewise, there was no condition $\times$ time interaction for the tension, depression, anger, vigour or confusion subscale $(P>0.05)$. The fatigue subscale trended towards more fatigue during the NER condition and less during the control condition but did not reach statistical significance $(F=3 \cdot 61 ; P=0 \cdot 06)$.

\section{Discussion}

The present study provides initial evidence that restricting night-time eating may promote a short-term reduction in total energy intake in a young male population. Our original hypothesis was thus correct. Several findings from the present study are given brief attention below.

First, daily energy intake was approximately $1000 \mathrm{~kJ}$ lower during the NER condition. This level of energy reduction is modest but similar to other 'small-changes' approaches ${ }^{(5,23)}$ that may be effective for body weight maintenance. We observed that a potential advantage of the NER intervention to reduce energy intake is its simplicity. There were no gadgets or record keeping and the intervention was simple to understand and implement. Second, the participants reported modestly higher levels of pre-breakfast hunger during the NER condition than during the control condition. While there was no difference in average energy intake during morning time (breakfast) between conditions (data not shown), it is unclear how the increase in pre-breakfast hunger would influence subsequent hunger and energy intake if an NER intervention was followed long term. Third, during the NER condition,

Table 3. Mood scores by condition

(Mean values and standard deviations)

\begin{tabular}{|c|c|c|c|c|c|c|c|c|c|c|}
\hline & \multicolumn{4}{|c|}{ Night-time eating restriction (n 27) } & \multicolumn{4}{|c|}{ Control condition (n 27) ${ }^{\star}$} & \multirow[b]{3}{*}{$\mathrm{F \dagger}$} & \multirow[b]{3}{*}{$P \dagger$} \\
\hline & \multicolumn{2}{|c|}{ Pre } & \multicolumn{2}{|c|}{ Post } & \multicolumn{2}{|c|}{ Pre } & \multicolumn{2}{|c|}{ Post } & & \\
\hline & Mean & SD & Mean & SD & Mean & SD & Mean & SD & & \\
\hline Total mood score & $13 \cdot 7$ & $16 \cdot 2$ & $14 \cdot 7$ & 20.7 & $16 \cdot 6$ & $25 \cdot 4$ & $12 \cdot 2$ & $16 \cdot 5$ & 0.47 & 0.494 \\
\hline Tension & $6 \cdot 6$ & $3 \cdot 2$ & $6 \cdot 8$ & $2 \cdot 7$ & $6 \cdot 7$ & 3.7 & $6 \cdot 6$ & 3.2 & 0.02 & 0.882 \\
\hline Depression & $5 \cdot 3$ & $5 \cdot 2$ & 4.9 & 4.9 & $5 \cdot 7$ & $6 \cdot 4$ & 4.3 & 4.0 & 0.25 & 0.619 \\
\hline Anger & 4.7 & 4.7 & 4.9 & $4 \cdot 8$ & 4.7 & $6 \cdot 4$ & $4 \cdot 1$ & $4 \cdot 3$ & $0 \cdot 13$ & 0.722 \\
\hline Vigour & 17.4 & 4.5 & $17 \cdot 0$ & $5 \cdot 3$ & $16 \cdot 8$ & $5 \cdot 4$ & $16 \cdot 3$ & $5 \cdot 5$ & 0.01 & 0.941 \\
\hline Fatigue & $6 \cdot 2$ & $4 \cdot 2$ & $7 \cdot 3$ & $5 \cdot 2$ & $8 \cdot 1$ & $5 \cdot 7$ & $5 \cdot 7$ & $4 \cdot 2$ & $3 \cdot 61$ & 0.060 \\
\hline Confusion & 6.5 & $2 \cdot 9$ & 6.5 & $2 \cdot 9$ & 6.9 & $3 \cdot 2$ & 6.5 & $3 \cdot 2$ & $0 \cdot 10$ & 0.752 \\
\hline
\end{tabular}

* No within-group difference was observed for the night eating restriction or control condition.

$\dagger F$ and $P$ values represent a condition $\times$ time interaction. 
the participants lost a small amount of body weight, and during the control condition, they gained a small amount of body weight. In conjunction with the average decrease in energy intake during the NER condition, these data suggest that the decreased energy intake during the NER intervention partially explained the slight weight loss. However, we note that the gain in weight during the control condition is surprising given that there was no difference in energy intake between the lead-in and control conditions. Therefore, we cannot rule out the possibility that there was under-reporting of energy intake or that some other study factor influenced energy intake, particularly during the control condition.

The major strength of the present study is that it experimentally examined the influence of NER on energy intake and body weight. Using a cross-sectional design or performing a secondary analysis, others have suggested that night-time/ late-night eating is positively associated $(r$ 0.14) with total daily energy intake in young/middle-aged adults ${ }^{(7)}$, influences diet and body weight in college-aged students ${ }^{(9)}$ and is associated with BMI and obesity ${ }^{(24,25)}$. On the other hand, Sullivan et al. ${ }^{(26)}$ reported that in rhesus monkeys, energy consumption at night did not correlate well with body weight gain. In a study related to the present investigation, Waller et al. ${ }^{(27)}$ studied the effects of a post-dinner snack intervention in overweight or obese 'night snackers' over 4 weeks. They showed that their intervention resulted in less energy intake of approximately $1561 \mathrm{~kJ} / \mathrm{d}$ than in the control group ${ }^{(27)}$. The implication of their findings was that minimising nighttime snacking may result in lower overall energy intake in that sample. However, it is difficult to compare the Waller et $a l{ }^{(27)}$ results with those of the present study, as the present study did not exclusively use overweight or obese subjects and did not provide snacks. Nevertheless, the present study appears to support other related studies of the potential effect of NER on energy intake and weight management outcomes.

Notable caution should be exercised when interpreting these data. First, the NER and control conditions each lasted for a period of only 2 weeks. The present study provides no information regarding the long-term effectiveness, practicality or compensatory effects that may occur over a longer period of time. Second, though being a student was not a requirement for participation, all participants in the final sample were university students. This prevents the broad generalisation of the results of the study. In addition, the participants in the present study were excluded for alcohol use. Other studies have shown that in young adults classified as 'current drinkers', increasing alcohol consumption (drinks/d) may be associated with higher energy intake ${ }^{(28)}$. Furthermore, in drinkers, it has been shown that alcohol consumption tends to increase during the evening hours ${ }^{(7)}$. Therefore, the present study does not generalise to a drinking population. Additional studies of the effect of NER in broader populations, including obese adults, children or families, may be particularly beneficial.

In conclusion, compared with the control condition, 2 weeks of NER resulted in a significant difference in energy intake and body weight in healthy young men. Therefore, these data provide initial proof of concept for NER. If NER proves sustainable in the long term, it may be a promising tool to prevent weight gain in this population sample. However, examination of the long-term effectiveness of NER and application to additional sample populations are needed.

\section{Acknowledgements}

There is no external funding source to report. The authors' contributions are as follows: J. D. L. conceived the study design with significant input from E. C., B. W. B. and L. A. T; E. C. L. coordinated the data recruitment and collection; L. A. T. provided additional statistical input; J. D. L. was the primary author of the manuscript with input and critical review from E. C., B. W. B. and L. A. T. There are no conflicts of interest to report.

\section{References}

1. Mozaffarian D, Hao T, Rimm EB, et al. (2011) Changes in diet and lifestyle and long-term weight gain in women and men. $N$ Engl J Med 364, 2392-2404.

2. Gropper SS, Simmons KP, Connell LJ, et al. (2012) Changes in body weight, composition, and shape: a 4-year study of college students. Appl Physiol Nutr Metab 37, 1118-1123.

3. Racette SB, Deusinger SS, Strube MJ, et al. (2008) Changes in weight and health behaviors from freshman through senior year of college. J Nutr Educ Behav 40, 39-42.

4. Rodearmel SJ, Wyatt HR, Stroebele N, et al. (2007) Small changes in dietary sugar and physical activity as an approach to preventing excessive weight gain: The America on the Move Family Study. Pediatrics 120, e869-e879.

5. Hill JO (2009) Can a small-changes approach help address the obesity epidemic? A report of the Joint Task Force of the American Society for Nutrition, Institute of Food Technologists, and International Food Information Council. $\mathrm{Am}$ J Clin Nutr 89, 477-484.

6. Wansink B (2004) Environmental factors that increase the food intake and consumption volume of unknowing consumers. Annu Rev Nutr 24, 455-479.

7. de Castro JM (2004) The time of day of food intake influences overall intake in humans. J Nutr 134, 104-111.

8. Thomson M, Spence JC, Raine K, et al. (2008) The association of television viewing with snacking behavior and body weight of young adults. Am J Health Promot 22, 329-335.

9. Nelson MC, Kocos R, Lytle LA, et al. (2009) Understanding the perceived determinants of weight-related behaviors in late adolescence: a qualitative analysis among college youth. J Nutr Educ Behav 41, 287-292.

10. Stunkard AJ, Grace WJ \& Wolff HG (1955) The night-eating syndrome; a pattern of food intake among certain obese patients. Am J Med 19, 78-86.

11. Colles SL, Dixon JB \& O'Brien PE (2007) Night eating syndrome and nocturnal snacking: association with obesity, binge eating and psychological distress. Int $J$ Obes 31, $1722-1730$

12. Atkinson G, Fullick S, Grindey C, et al. (2008) Exercise, energy balance and the shift worker. Sports Med 38, 671-685.

13. Hager R, George JD, LeCheminant JD, et al. (2012) Evaluation of a university general education health and 
wellness course delivered by lecture or online. Am J Health Promot 26, 263-269.

14. Stunkard A, Berkowitz R, Wadden T, et al. (1996) Binge eating disorder and the night-eating syndrome. Int $J$ Obes Relat Metab Disord 20, 1-6.

15. Hanlon B, Larson MJ, Bailey BW, et al. (2012) Neural response to pictures of food after exercise in normalweight and obese women. Med Sci Sports Exerc $\mathbf{4 4}$, $1864-1870$

16. Striegel-Moore RH, Franko DL, Thompson D, et al. (2006) Night eating: prevalence and demographic correlates. Obesity 14, 139-147.

17. Johnson RK (2002) Dietary intake - how do we measure what people are really eating? Obes Res 10, 63S-68S.

18. Bailey BW, Tucker LA, Peterson TR, et al. (2001) Test-retest reliability of body fat percentage results using dual energy X-ray absorptiometry and the Bod Pod. Med Sci Sports Exerc 33, 174 S.

19. Maddalozzo GF, Cardinal BJ \& Snow CA (2002) Concurrent validity of the BOD POD and dual energy X-ray absorptiometry techniques for assessing body composition in young women. J Am Diet Assoc 102, 1677-1679.

20. Kral TV, Roe LS \& Rolls BJ (2004) Combined effects of energy density and portion size on energy intake in women. $A m J$ Clin Nutr 79, 962-968.
21. Lemmens SG, Martens EA, Kester AD, et al. (2011) Changes in gut hormone and glucose concentrations in relation to hunger and fullness. Am J Clin Nutr 94, 717-725.

22. Nyenhuis DL, Yamamoto C, Luchetta T, et al. (1999) Adult and geriatric normative data and validation of the profile of mood states. J Clin Psychol 55, 79-86.

23. Stroebele N, de Castro JM, Stuht J, et al. (2009) A smallchanges approach reduces energy intake in free-living humans. J Am Coll Nutr 28, 63-68.

24. Lissner L, Lindroos AK \& Sjostrom L (1998) Swedish obese subjects (SOS): an obesity intervention study with a nutritional perspective. Eur J Clin Nutr 52, 316-322.

25. Baron KG, Reid KJ, Kern AS, et al. (2011) Role of sleep timing in caloric intake and BMI. Obesity 19, 1374-1381.

26. Sullivan EL, Daniels AJ, Koegler FH, et al. (2005) Evidence in female rhesus monkeys (Macaca mulatta) that nighttime caloric intake is not associated with weight gain. Obes Res 13, 2072-2080.

27. Waller SM, Vander Wal JS, Klurfeld DM, et al. (2004) Evening ready-to-eat cereal consumption contributes to weight management. J Am Coll Nutr 23, 316-321.

28. Breslow RA, Guenther PM, Juan W, et al. (2010) Alcoholic beverage consumption, nutrient intakes, and diet quality in the US adult population, 1999-2006. J Am Diet Assoc 110, $551-562$. 